ACTA UNIVERSITATIS LODZIENSIS

Folia Litteraria Romanica 16, 2021

https://doi.org/10.18778/1505-9065.16.07

\author{
Verónica Rodriguez Ferreiro* \\ Université de Saint-Jacques de Compostelle \\ (iD) https://orcid.org/0000-0002-5845-4449 \\ veronica.rodriguez.ferreiro0@usc.es
}

\title{
De quelques proverbes qui font l'éloge de la femme
}

\begin{abstract}
RÉSUMÉ
Le proverbe reflète la réalité sociale et les croyances populaires. Il peut être envisagé dans le cadre d'une parémiologie comparée, de sorte que nous entreprenons cette étude comme une contribution au dictionnaire phraséologique français-espagnol-galicien. Nous avons eu recours à un ensemble de parémies en langue française, appartenant à différentes époques et sans nous limiter à une perspective eurocentriste, en nous basant sur une culture francophone, comme ensemble linguistique, culturel et sociologique de la francophonie. Le champ de travail a été restreint aux proverbes ayant trait d'une certaine manière à l'amour, au sens large, associé à la figure féminine, comme source d'épanouissement, de discernement, d'opulence, dans son rôle de mère irremplaçable ou comme l'égale de l'homme. Il n'y est question que de ceux qui en font l'éloge ou desquels il en ressort un aspect positif, au moins dans l'une de leurs possibles interprétations, contrairement à ceux, bien plus habituels et nombreux, qui en offrent une image négative.
\end{abstract}

MOTS-CLÉS - proverbe(s), parémiologie, lexicographie, femme(s), féminisme

\section{Regarding Some Proverbs that Praise Women}

\section{SUMMARY}

The proverb reflects social realities and popular beliefs. It can be considered within the framework of a comparative paremiology, so that we undertake this study as a contribution to the FrenchSpanish-Galician phraseological dictionary. We have resorted to whole paroemias in the French languages, belonging to different times and without the limitation of an Eurocentric perspective, basing ourselves on this Francophone culture, as a linguistic, cultural and sociological ensemble of the Francophonie. This field of work has been restricted to proverbs relating in some way to love, in the broad sense, associated with the female figure, as a source of fulfilment, of discernment, of opulence, in her role as irreplaceable mother or as an equal to the man. It is only a question of those which praise or transmit a positive aspect of women, at least in one of their possible interpretations, unlike the others, much more usual and numerous, which offer a negative image.

KEYWORDS - proverb (s), paremiology, lexicography, woman, feminism

* Travail partiellement réalisé dans le cadre du projet de recherche FFI2017-85141P (FEDER/Ministerio de Ciencia, Innovación y Universidades/AEI). 


\section{Introduction}

Les proverbes ont longtemps été perçus comme une manifestation linguistique mineure et, en conséquence, repoussés de toute analyse et ravalés au rang des procédés stylistiques. Cependant, ils ont toujours suscité de la fascination, en raison de leur nature contradictoire. À leur apparente simplicité s'oppose leur capacité d'adaptation à toutes sortes de circonstances. Et leur présumée naïveté est contestée par leur facilité à véhiculer une image, généralement sur la nature ou les animaux, de la réalité sociale environnante, afin de transmettre un message à caractère global. Or, ces dernières années se sont développés bon nombre d'études parémiologiques ${ }^{1}$.

Les proverbes ont su consolider la valeur de leurs caractéristiques, non seulement phonétiques et stylistiques, mais aussi sémantiques et morphosyntaxiques, de même que leur importance pour l'histoire socioculturelle des peuples. En effet, si bon nombre d'anciens proverbes se rapportent à la météorologie, c'est parce que la prévision du temps était autrefois essentielle pour les travaux agricoles ou la pêche, seuls moyens de subsistance de la société rurale. De même, on explique un grand nombre de proverbes préconisant l'austérité dans le nord et l'est de la France, de par l'influence du protestantisme. Ainsi, considérés comme « le fait de langage par excellence, qui reflète les réalités sociales à travers la symbolique de ses constituants nominaux, qui en conforment la charpente »(Claver Zouogbo, $2009: 18$ ), ils représentent des valeurs propres à chaque culture. Et compte tenu de l'actuelle globalisation et de l'importance des échanges interculturels, nous considérons qu'il serait intéressant d'encourager les études de parémiologie comparée ${ }^{2}$ entre les langues française, espagnole et galicienne, dont les interactions restent vivaces.

Nous avons regroupé cet intérêt croissant des études linguistiques et lexicographiques envers les parémies avec le discours social dominant du mouvement féministe, en nous penchant sur les proverbes qui font l'éloge de la femme, au moins dans l'une de leurs possibles interprétations. Cependant, malgré la proximité existante entre les trois langues, l'équivalence pleine entre les proverbes ne se produit que très rarement, compte tenu des nuances et des modes d'expression qui diffèrent d'une langue à l'autre. Par conséquent, la notion d'équivalence doit être conçue dans son sens le plus large, en raison des particularités du patrimoine culturel et linguistique de chacune de ces communautés linguistiques.

${ }^{1}$ On citera pour la langue française celles de Rodegem, Greimas, Anscombre, Arnaud, Kleiber, Norrick ou Rey, entre autres. En Espagne, les centres de recherches phraséologiques favorisent les études menées par Corpas Pastor et Sevilla Muñoz en espagnol, ainsi que celles de Conde Tarrio et de Gonzalez Rey en galicien.

${ }^{2}$ Cette brève approche à ce qui pourrait être un dictionnaire de parémies françaises-espagnoles-galiciennes, pourvu de quelques informations additionnelles au caractère encyclopédique et relatives aux origines, éléments rhétoriques et autres, est disponible sur le site https://refrans-proverbes.com que nous alimenterons au fur et à mesure de nos progrès. 


\section{L'intérêt de la recherche sur les proverbes et autres unités phraséologiques}

Nous souhaitons tout d'abord faire une réflexion sur les dictionnaires phraséologiques français-espagnol-galicien. Toutefois, les limites de cet article nous contraignent à ne présenter qu'un bref échantillon de quelques unités, sélectionnées parmi les proverbes et autres expressions apparentées. Nous espérons tout de même contribuer à l'enrichissement de la conservation de parémies pour les locuteurs de ces langues. Il devrait également en découler une réflexion générale sur celles-ci et, en particulier, sur celles dont l'usage a diminué, en raison de la distance existant entre les modes de vie contemporains et les références employées. En effet, même si en Galice, pour l'instant, le proverbe ne constitue pas encore un vestige archaïsant de la langue, car il est vivant et fonctionnel pour un nombre considérable de locuteurs (Conde Tarrio, 1998 : 62), il n'en va pas de même pour l'espagnol, et moins encore pour la langue française, dont « le fond est plus archaïque et surtout moins usité » (Montreynaud, Pierron, Suzzoni, 1989: 5).

Cette approche vise principalement les sujets parlants de ces trois langues, afin qu'ils puissent produire et décoder des textes oraux et écrits en français, espagnol et galicien. La connaissance et l'emploi des proverbes est un indicateur de la maîtrise de la langue, dès lors qu'ils impliquent des procédés linguistiques fossilisés, étrangers à la langue actuelle et qu'ils représentent, dans une grande mesure, le patrimoine culturel d'un peuple, sa manière de concevoir la vie, la nature, l'homme, Dieu, etc. C'est pour cette raison qu'il s'avère extrêmement compliqué de trouver des parémies identiques dans les différentes langues, même si, comme dans ce cas, le français, l'espagnol et le galicien appartiennent à la même famille romane et, à l'origine, au même milieu culturel de l'Europe occidentale. En réalité, l'identité totale ne se produit que très rarement car, bien que l'idée transmise puisse être analogue, il existe normalement des nuances additionnelles dans chacune des langues et la manière de s'exprimer répond normalement à des procédés lexicaux, syntaxiques, sémantiques et rhétoriques différents. Aussi, généralement, nous faut-il consentir à des équivalences partielles qui, tantôt se rapprochent davantage de la pensée exprimée, tantôt de la forme utilisée et ce, sans nous limiter aux proverbes au sens strict du terme, mais en ayant recours à un ensemble d'énoncés phraséologiques.

Dans ce sens, nous nous situons dans le courant exocentriste promu par Ladmiral (1999 : 142) dans le champ de la traduction, de sorte que les unités mises en relation présentent, selon notre critère, et malgré les possibles différences de forme ou même de formulation du contenu, une pensée commune et permettent, par là même, une traduction formelle, directement dérivée de l'esprit des belles infidèles de Gilles Ménage (1654 : 324). Nous citerons, à titre d'exemple, le proverbe Une hirondelle ne fait pas le printemps, face à Una golondrina no hace verano, ou Unha andoriña non fai verán ; même si en Espagne et en Galice le proverbe mentionne l'été, les hirondelles arrivent aussi au printemps. Toute autre approche interlinguistique serait, d'après nous, faussée. 
Ainsi, les parémies favorisent la comparaison des similitudes et des différences socioculturelles et peuvent contribuer, par là même, à briser bon nombre de préjugés, stéréotypes et barrières existantes vis-à-vis des autres peuples, et qui fréquemment se reflètent dans ce genre d'unités phraséologiques.

Dans ce sens, et compte tenu de l'actuel contexte social de forte revendication de la figure féminine, nous avons décidé de sélectionner des proverbes faisant, d'une certaine manière, l'éloge de la femme, au moins dans l'une de ses interprétations, afin de contribuer à la thématique de ce volume collectif sur l'amour et, surtout, par opposition à d'autres proverbes, bien plus fréquents, qui renferment des micromachismes ${ }^{3}$.

\section{La famille du proverbe}

En premier lieu, il convient de donner un bref aperçu sur le concept de proverbe. En l'absence d'une délimitation claire, les termes proverbe en français et refrán en espagnol et en galicien sont couramment rapprochés d'autres expressions apparentées, ayant une signification proche et avec lesquelles ils partagent certaines caractéristiques, malgré le fait que chacune ait des traits distinctifs qui lui sont propres, et qui la différencient des autres en général et du proverbe en particulier.

D'après Zuluaga Ospina (1975), toutes ces combinaisons de el acervo lingüistico del hablante ont pour caractéristique fondamentale leur Starrheit ou Frozenness, c'est-à-dire leur caractère figé, d'où leur dénomination en tant qu'expressions fixes ou unités de texte répété. Cette fixation peut se manifester de diverses manières, comme par le biais de «l'inaltérabilité de l'ordre des composants, l'invariabilité de certaines catégories grammaticales, le caractère non modifiable de l'inventaire des composants ou non remplaçable de ces éléments composants » Zuluaga Ospina (1975 : 226).

Parmi ces expressions figées, on distingue, d'une part, les énoncés phraséologiques, " communément appelés proverbes, adages, parémies, sentences, maximes, wellerismes, dialogismes, équivalents de syntagmes verbaux » et, d'autre part, les unités phraséologiques qui incorporent un prédicat complet mais qui " no constituyen por sí mismas un enunciado completo o minitexto » (Zuluaga Ospina, 1977: 631). Dans cette catégorie, Corpas Pastor (1996 : 88) y insère également les locutions, considérées comme une « unidad fraseológica del sistema de la lengua con los siguientes rasgos

\footnotetext{
3 Nous en fournissons quelques exemples : "Une bonne femme est une mauvaise bête ", "Une femme ne cèle que ce qu'elle ne sait pas », «Qui femme a, noise a », « Le cerveau de la femme est fait de crème de singe et de fromage de renard », "Ce n'est rien, c'est une femme qui se noie » (Quitard, 1961 : 401).
} 
distintivos : fijación interna, unidad de significación y fijación externa », bien que « no constituyen enunciados completos y, generalmente, funcionan como elementos oracionales ». Somme toute, les dénommées locuciones clausales, qui s'encadrent dans les locuciones oracionales (Corpas Pastor 1996 : 109), disposent effectivement d'une structure phrastique. C'est pour cette raison que, parfois, on les apparente aux parémies comme dans le cas de Rien n'est meilleur qu'une bonne femme, mais aussi parce qu'occasionnellement elles peuvent avoir un caractère sentencieux, comme dans garder les apparences, guardar las apariencias ou gardar as aparencias; ou être reliées au domaine populaire, comme pour faire bon (ou mauvais) marché, hacer buena (o mala) compra, ou facer boa (ou mala) feira; ou disposer d'une structure rythmique ; ou encore présenter une disposition bimembre, comme dans botar lume pola boca. García Page (2008 : 36) insiste sur la proximité de ces deux notions, mais prévient aussi sur le fait que «muchos refranes de actual estructura unimembre, incluso, bimembre, como muchas construcciones comparativas deben describirse como locuciones » (2008: 9).

\section{Les traits communs aux énoncés phraséologiques}

Les énoncés phraséologiques présentent donc une série de caractéristiques qu'ils partagent partiellement entre eux, tout en se différenciant par d'autres aspects propres à chacun, de sorte que, comme l'affirme Anscombre (1994 : 95), " on sent confusément qu'ils ne sont pas synonymes, sans pouvoir cependant étayer cette intuition ». Malgré leurs différences, Arnaud (1992), conformément aux considérations d'autres experts, tels que Thérond (1972), Rodegem (1973), Gross (1982), Norrick (1985), Kleiber (1988), en conclut que l'ensemble des énoncés phraséologiques partagent jusqu'à sept caractéristiques, dont les quatre premières sont généralement considérées restrictives ou obligatoires :

1. Le sens référentiel : l'existence d'une convention référentielle qui rattache l'énoncé phraséologique, en tant que signe, à son référent.

2. Le sens fonctionnel : la fonction communicative, littérale ou figurée, vis-àvis d'une situation déterminée.

3. Le sens propositionnel : l'expression d'un précepte d'ordre général relatif au comportement humain vis-à-vis de son entourage.

4. La fonction didactique : l'exposé d'une brève leçon de morale ou sagesse.

5. Le caractère traditionnel : l'origine ancienne ou dérivée d'un précurseur antique. mixte.

6. Le caractère métaphorique : l'interprétation littérale, métaphorique ou

7. Les structures prototypiques : la réitération de certaines structures récurrentes. 


\section{Les traits distinctifs des énoncés phraséologiques}

Toutefois, malgré ces qualités communes, relatives aux énoncés phraséologiques et qui motivent la proximité entre les différentes parémies, les énoncés présentent naturellement aussi des éléments distinctifs. Malgré quelques nuances divergentes, la plupart des chercheurs, tels que Lazaro Carreter (1980), Anscombre (1994), Corpas Pastor (1996), Alvarez de la Granja (2003) ou Claver Zouogbo (2009), conviennent de caractériser chacun d'eux de la manière suivante :

- L'adage est un énoncé à caractère juridique, qui se distingue aussi par son origine ancienne et attestée par l'expérience.

- L'aphorisme exprime une idée morale ou philosophique, succinctement résumée et généralement attribuée à un auteur.

- L'apophtegme lance un message exemplaire, bref et ingénieux, exprimé à travers des personnages représentatifs d'une classe ou d'une catégorie sociale spécifique, et dont la paternité est attribuée à une personne d'autorité.

- La citation constitue un corrélat non anonyme d'un énoncé lexicalisé à valeur spécifique, que l'on cite.

- Le dialogisme renferme un message né de l'expérience, mais sans valeur de vérité universelle, exposé de manière comique, à travers une structure phrastique bimembre ou trimembre, ou sous l'apparence d'un microtexte narratif ou dialogué.

- La maxime équivaut à un précepte, une norme morale ou un modèle de conduite exprimé de manière brève et concise.

- Le slogan renferme un message personnalisé et contextualisé, dans un but publicitaire ou didactique.

- Le truisme proverbial expose un principe d'apparente banalité, exprimé à travers des formules consacrées, répétitives ou tautologiques, mais jamais de manière métaphorique.

- Le wellerisme révèle une dénonciation de certains défauts, faite de manière ironique et non didactique à travers un personnage de fiction.

- Le proverbe est un énoncé qui renferme une valeur de vérité générale, ayant une signification descriptive, situationnelle et pragmatique. À noter que le terme français proverbe renferme les deux termes espagnols et galiciens refrán et proverbio, dont le deuxième se distingue du premier du fait qu'il est un énoncé sentencieux, adopté par la communauté, transmis par écrit, ce qui en assure son origine et lui octroie un ton plus grave de par sa source cultivée.

- Le dicton consiste en un proverbe encadré dans une thématique météorologique, géographique ou relative au travail.

\section{La délimitation des caractéristiques des proverbes}

La distinction entre les différents termes est, comme nous venons de le voir, très nuancée. Aussi, dans le but de délimiter les caractéristiques essentielles et inhérentes aux proverbes, de sorte à pouvoir les identifier comme tels et à les 
différencier de toute autre construction similaire, Arnaud (1992) a établi six critères spécifiques. Ils ont été ultérieurement repris de manière plus exhaustive par Anscombre (1994) et, de manière générale, Corpas Pastor (1996) et Alvarez de la Granja (2003) les soutiennent également :

- La lexicalité, soit le caractère codé et préconstruit ;

- L'autonomie syntaxique, c'est-à-dire la possibilité d'être énoncé de manière naturelle, sans modification, ni incipit ;

- L'autonomie textuelle, donc son indépendance vis-à-vis du discours ;

- La valeur de vérité générale ou le côté prescriptif, qui traduit l'indépendance du cadre spatio-temporel subordonné au contexte de l'énonciation ;

- L'anonymat, conçu en tant qu'appartenance au fonds commun linguistique et culturel pour avoir perdu la paternité ;

- Le côté métaphorique ou imagé. Il s'agit là d'un critère controversé, en raison de la possible double interprétation (littérale et métaphorique), et de son rattachement aux comportements humains.

Après avoir délimité les caractéristiques de l'ensemble des parémies et des proverbes en particulier, nous proposons de nous pencher sur quelques exemples de proverbes, peu nombreux, qui font l'éloge de la femme et en exaltent quelques-unes de ses qualités, que ce soit au moins dans l'une de leurs possibles interprétations, ce qui parfois contrevient à l'interprétation habituelle, les croyances populaires ou les préjugés sociaux.

\section{Les proverbes qui font l'éloge de la femme : quelques considérations formelles et sémantiques}

Dans cette thématique de la mise en valeur des qualités de la femme, nous avons relevé des exemples de plusieurs types : à savoir, la femme comme source d'épanouissement, de discernement, d'opulence, dans son rôle de mère irremplaçable ou comme l'égale de l'homme.

Le manque de recherches en matière de parémiologie comparée entre les langues française, espagnole et galicienne, de même que la difficulté pour trouver des parémies favorables à la condition féminine, nous ont contraint à utiliser des répertoires francophones de tous genres. Le corpus, en apparence hybride, répond à ces besoins. Le tri se réalise d'après les résultats obtenus; le point de connexion étant la thématique de la valorisation de la femme, par opposition à la grande masse de proverbes où elle est stigmatisée. Nous avons eu recours à un ensemble de parémies en langue française, au-delà de la perspective eurocentriste, en nous basant sur une culture francophone, comme ensemble linguistique, culturel et sociologique de la francophonie, conformément à la définition de Michel Têtu (1992). De même, nous y avons inclus des proverbes anciens, dans la volonté de les sortir de l'oubli, dans un but à la fois linguistique et social. 


\subsection{La femme comme source d'épanouissement}

6.1.1. a) Celui qui a avec lui une femme, Dieu est avec lui (Ould Ebnou, Ould Mohameden, $2008: 26$ ).

b) Celui qui a la femme de son côté, à Dieu avec lui (Ould Ebnou, Ould Mohameden, $2008: 134)^{4}$.

Origine : Ce proverbe français est employé essentiellement en Mauritanie, bien qu'on y constate une influence assez probable des Proverbes bibliques (Les Proverbes de Salomon : XVIII, 12). Il brise un tant soit peu la conception occidentale que nous avons de la valorisation de la femme dans les pays musulmans.

Signification : L'idée transmise est que la présence d'une femme dans la vie de l'homme lui permet d'atteindre la plénitude, dès lors qu'elle va de pair avec le soutien du Seigneur. Cette caractéristique est attribuée à toute les femmes, étant donné qu'il ne s'y établit aucune condition.

Structure et éléments rhétoriques : Les deux variantes présentent des structures dissemblables. La première (a) met en avant le complément d'objet pourvu d'une relative et lui accorde ainsi une plus grande emphase, tandis que la deuxième (b) commence par une proposition relative ayant la fonction de sujet. À noter la répétition du pronom personnel lui.

\subsubsection{Quien tiene mujer, tiene lo que ha menester (Instituto Cervantes).}

Équivalence : Il n'existe pas d'équivalence pleine entre le proverbe français et les proverbes espagnols et galiciens, qui eux sont équivalents. Le proverbe français assimile la compagnie de la femme à la protection de Dieu, alors que le proverbe espagnol est dépourvu de toute connotation religieuse (de même que le proverbe galicien ci-après).

Signification : La compagnie d'une épouse est la meilleure garantie pour l'homme de vivre heureux, puisqu'elle représente tout ce dont il peut avoir besoin, entendu dans son sens le plus large.

Structure et éléments rhétoriques : Phrase complexe débutant par une subordonnée relative en fonction de sujet. La mémorisation est facilitée grâce à la répétition de tiene et à la rime finale en -er.

\subsubsection{Quen ten muller, ten o que ha mester (DEGC, s.v. femme; Moreiras} Santiso, 1978 : 77; Sesto López, 1976 : 41).

Équivalence : Le proverbe galicien montre une équivalence totale avec le proverbe espagnol, tant sur le fond, que sur la forme, mais n'a qu'une équivalence

\footnotetext{
${ }^{4}$ Nous avons mis en gras les proverbes en langue française et en italiques ceux en langue castillane et galicienne.
} 
partielle avec le proverbe français, qui lui a recours à des références religieuses pour rapprocher la figure féminine de celle du Seigneur. À noter que, contrairement à d'autres, tous ces proverbes recueillent cette capacité comme inhérente à la femme, sans conditions de caractère.

Signification : La compagnie d'une épouse est garante de bonheur, sans prérequis.

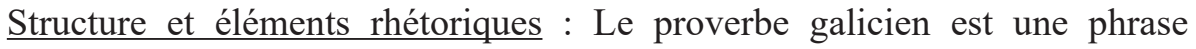
complexe ayant pour sujet une subordonnée relative, conformément à la structure proverbiale prototypique. Le pronom relatif sujet quen possède un caractère indéfini et a le sens de n'importe qui, quiconque.

\subsection{La femme est l'égale de l'homme}

\subsubsection{La femme est la moitié de l'homme (Quitard, $1861: 61$ ).}

Origine : Quitard (1861:87) le met en relation avec le verset biblique «C'est pourquoi l'homme quittera son père et sa mère, et s'attachera à sa femme; et ils seront deux dans une seule chair » (Genèse : II, 24). Il pourrait dériver du latin Dimidia pars hominis.

Signification : Malgré l'avis populaire, ce proverbe ne doit pas être interprété dans le sens que la femme a la moitié de la valeur de l'homme, mais qu'elle contribue dans une très grande mesure à son développement personnel et professionnel; elle agit comme son associée. Si on en revient à une signification biblique, le proverbe rappelle que l'homme et la femme constituent les deux moitiés d'une seule et même personne.

Structure et éléments rhétoriques : D'un point de vue syntaxique, il s'agit d'une phrase simple à caractère sentencieux.

\subsubsection{La mujer es la mitad del hombre (Etxabe Díaz, 2012 : 241).}

Équivalence : Nous avons une équivalence pleine tant sur le fond que sur la forme.

Signification : L'interprétation habituelle est tendancieusement faussée, dès lors qu'elle suggère que la valeur de la femme est à diviser par deux visà-vis de celle de l'homme. Au contraire, ce proverbe met en évidence que l'homme est redevable envers la femme de la moitié de ses succès personnels et professionnels.

Structure et éléments rhétoriques : La forme syntaxique de ce proverbe correspond à celle d'une phrase simple copulative, d'une remarquable simplicité et brièveté, ce qui contribue à faciliter à la mémoriser. 
6.2.1.2. a) A muller é a metade do home (Moreiras Santiso, 1978 : 77).

b) A muller é a mitá do home (Sesto López, 1976 : 34).

Équivalence : L'équivalence est pleine, tant sur le fond, que sur la forme.

Signification : Souvent, ce proverbe galicien est sujet à une interprétation erronée, en raison d'une mentalité sexiste qui estime qu'un homme a le double de la valeur d'une femme.

Structure et éléments rhétoriques : Le proverbe présente la forme syntaxique d'une phrase simple copulative. Quant aux éléments rhétoriques, nous ne pouvons mentionner que la simplicité et la brièveté, qui participent à mémorisation et à transmission de la sentence, de même que l'allitération du phonème nasal bilabial qui en accroît la sonorité.

\subsection{La femme comme source de discernement}

\subsection{1. a) La barbe dit le jour ce que la natte dit la nuit (Desalmand, Stalloni, $2013: 62$ ). \\ b) La barbe exécute le jour la volonté de la tresse la nuit (Ould Ebnou, Ould Mohameden, 2008 : 136).}

Origine : Les deux variantes remontent au XVIème siècle.

Signification : La barbe représente l'homme, alors que la tresse ou la natte font allusion à la femme qui durant très longtemps, et même encore aujourd'hui dans certaines sociétés ou cercles sociaux, n'a pu s'exprimer librement qu'en privé. De même, c'est lorsqu'elle est seule avec son mari, ce qui ne se produit généralement que le soir, qu'elle peut faire part de son avis et donc de ses conseils, dont l'habituelle pertinence pousse le mari à les adopter dès le lendemain. Il est intéressant de constater comment un proverbe, attesté depuis le XVIème siècle, témoigne de la participation indirecte des femmes dans les prises de décision, alors même qu'on leur refusait n'importe quelle intervention socialement visible.

Structure et éléments rhétoriques : Les deux proverbes renferment les métonymies barbe-homme et natte/tresse-femme. La seule différence syntaxique entre les deux variantes est que, dans le premier proverbe, le complément d'objet direct renferme une subordonnée relative en fonction de sujet, ce qui ne se produit pas dans le deuxième.

\subsubsection{La mujer que calla de día por la noche manda. (Cardona, 2013 : 99).}

Équivalence : Il n'y a pas d'équivalence dans l'expression, bien que l'idée transmise soit la même. En effet, la femme, qui se doit d'avoir une personnalité effacée en public, fait part de ses suggestions en privé, la nuit venue. On peut 
présupposer la pertinence de ses conseils, qui seront donc suivis par le mari, de sorte qu'on peut considérer que c'est elle qui prend les décisions.

Signification : La société impose à la femme un rôle secondaire en public, qui l'empêche de montrer ce qu'elle vaut. Malgré tout, elle intervient indirectement dans les prises de décision de son mari, qui reconnaît ainsi la pertinence de ses conseils.

Structure et éléments rhétoriques : Le sujet de la phrase renferme une subordonnée relative, qui permet la formation d'une structure parallèle avec un jeu d'opposition calla et día face à noche et manda.

\subsubsection{O consello da muller é pouco, mais o que non o sigue é un louco} (Moreiras Santiso, 1978 : 48 ; Sesto López, 1976 : 26).

Équivalence : Ce proverbe galicien n'a ici qu'une équivalence partielle, car outre la structure, on y perd aussi l'idée que les conseils de la femme sont transmis en catimini, dans l'intimité du foyer, pour maintenir le rôle secondaire, auquel l'oblige la société. Malgré tout, le proverbe reconnaît leur juste valeur, qualifiant d'insensés ceux qui ne les suivent pas.

Signification : Le proverbe proclame la haute valeur des conseils féminins et déclare fous ceux qui n'en tiennent pas compte.

Structure et éléments rhétoriques : Le proverbe se construit sur la base de deux propositions coordonnées par une conjonction adversative. Le sujet de la deuxième proposition est une subordonnée relative. On constate également la présence d'une rime consonantique entre pouco et louco qui participe de la mémorisation du proverbe dans l'esprit des locuteurs.

\subsection{La femme comme source d'opulence}

\subsubsection{Prenez des femmes, elles vous apporteront des richesses (Schipper, 2008 : 117).}

Origine : Le proverbe est originaire des régions francophones du Maghreb.

Signification : Le proverbe affirme que les aptitudes féminines pour travailler, administrer les biens, etc. garantissent à l'homme une bonne qualité de vie. Indirectement, il pourrait également suggérer que ces mêmes qualités manquent à l'homme, d'où le besoin de se marier exprimé au mode impératif. Il serait erroné de penser que ces richesses correspondent au paiement de la dot, étant donné qu'au Maghreb, contrairement aux coutumes occidentales, c'est l'homme qui apporte des biens à l'union matrimoniale.

Équivalence : Le proverbe est formé de deux propositions juxtaposées, dont la deuxième a une valeur causale. 
6.4.1.1. La mujer buena, de la casa vacía la hace llena. (De Barros, 1945 : 298).

Équivalence : Il s'agit d'une équivalence partielle, bien que l'idée transmise soit la même. Toutefois, contrairement au proverbe en français, celui-ci conditionne cette aptitude féminine au caractère bienveillant de la mujer buena. De plus, le proverbe espagnol ne fait pas allusion au mariage et s'applique de manière générale à toutes les femmes.

Signification : Les bonnes femmes ont la capacité de travailler et d'organiser leurs ressources de sorte à assurer le bien-être du foyer.

Équivalence : Le proverbe utilise une structure fossilisée avec une altération de l'ordre normal des mots à l'intérieur de la phrase. Au niveau rhétorique, on apprécie l'emploi de la métaphore hace llena la casa dans le sens de 'pourvoir au bien-être de la famille'. À noter l'allitération en [a] qui donne du rythme et favorise la mémorisation et transmission de la parémie.

\subsubsection{A boa muller a casa sabe encher (Lorenzo Fernández, 1983: 13).}

Équivalence : Le proverbe galicien présente une équivalence partielle vis-àvis du français, mais une équivalence totale avec le proverbe espagnol.

Signification : Le proverbe exprime la capacité de travail et d'organisation des femmes pour pourvoir aux besoins de leurs famille. À noter qu'on conditionne à nouveau cette capacité au caractère bienveillant de la femme.

Équivalence : Le proverbe est une phrase simple qui, pourtant, utilise une structure fossilisée avec une altération de l'ordre normal des mots à l'intérieur de la phrase. L'ellipse encher a casa se rapporte également à la capacité de la femme à assurer le bien-être de sa famille. Finalement, la rime [er] favorise la mémorisation de la parémie.

\subsection{La femme dans son rôle de mère irremplaçable}

\subsubsection{Qui lui a pris son père n'a rien pris, qui lui a pris sa mère n'a rien} laissé (Belamri, 1986 : 39).

Origine : Le proverbe est d'origine algérienne.

Signification : Le proverbe se présente sous une forme abstraite, dès lors qu'il fait référence à l'élément sapientiel. Il manque de références matérielles, celles qui renvoient au foyer, au profit des émotions. À souligner que l'influence paternelle y est tout à fait dépréciée, réduite à rien.

Structure et éléments rhétoriques : Nous avons une structure bimembre composée par deux propositions juxtaposées, à valeur d'opposition. Les deux séquences présentent la même organisation syntaxique, avec l'anaphore qui lui 
a pris. L'antithèse se produit moyennant la substitution de son père par sa mère et n'a rien pris par n'a rien laissé. De même, le recours à l'hyperbole permet de renforcer l'expression.

\subsubsection{1. a) Muerte del padre casa no deshace, pero sí la muerte de la madre. (Glowicka, 2007 : 270), (Fernandez Poncela, 2015 : 67). \\ b) Madre muerta, casa deshecha. (Glowicka, 2007 : 270), (Fernandez Poncela, $2015: 67)$.}

Équivalence : Le premier des proverbes espagnol présente une équivalence partielle sur le fond et sur la forme, tandis que le deuxième ne le fait que sur le sens. En effet, la variante a) transmet l'idée que la mort de la mère a des conséquences dévastatrices au sein de la famille, ce qui n'est pas le cas pour la mort du père, alors que la variante b) échappe à cet effet de contraste et se limite à déplorer les effets ravageurs qui suivent le décès de la femme.

Signification : Les deux proverbes transmettent l'idée que la mort de la mère entraîne la destruction du foyer en tant qu'unité familiale.

Structure et éléments rhétoriques : Le premier proverbe présente une structure bimembre et thématise le sujet de la mort, muerte del padre - muerte de la madre, qu'il place au début de chacune des propositions, reliées entre elles par le coordonnant adversatif pero. Les deux parties sont mises en parallèle, malgré le fait que casa no deshace soit remplacé par l'adverbe sí à valeur contrastive dans la deuxième partie. Les deux proverbes font usage de la même métaphore deshacer casa pour transmettre l'idée de destruction de l'unité familiale. Quant à la variante b), elle apparait comme un résumé de a), dont elle n'exprime que l'information significative exposée dans la deuxième partie. Sa brièveté, ainsi que la présence de la rime en - $a$ en garantissent une meilleure mémorisation et transmission.

\subsubsection{2. a) Desgraciados os fillos que quedan sen pai, pero máis desgraciados son os que quedan sen nai (Moreiras Santiso, 1978 : 63; Sesto López, 1976 : 43; Zamora Mosquera, 1972 : 79). \\ b) Morte de pai, casa non desfai, pero si morte de nai (Sesto Lopez, 1976 : 44).}

Équivalence : Le proverbe galicien a) est plus proche du français, non seulement quant au sens, mais aussi quant à l'expression parallèle et à sa configuration rhétorique, quoique différente. Quant à b), il existe une équivalence partielle, tant sur le fond que sur la forme, entre ce proverbe galicien, le premier proverbe espagnol et le proverbe français. En effet, bien que tous fassent allusion à la mort, celle-ci n'est explicite que dans le deux premiers, le français lui préférant une formule voilée moyennant le verbe prendre qui, naturellement, doit être interprété dans ce même sens. De même, en attribuant le rôle principal dans le foyer à la femme, on accorde la priorité au besoin de compter sur le soutien de 
la mère. À noter toutefois que le proverbe français est dépourvu des connotations matérielles de casa, pour privilégier les sentiments.

Signification : Le proverbe a) met en relief le rôle prépondérant de la mère. L'usage du mot fillo, au lieu de neno ou cativo, renforce l'idée que la figure de la mère est nécessaire tout au long de la vie, et pas seulement pendant l'enfance. Le proverbe b) met en évidence que le décès de la femme entraîne, de par son rôle essentiel, un cataclysme au sein de l'unité familiale.

Structure et éléments rhétoriques : L'organisation syntaxique de a) et b) se réalise au moyen d'une coordination adversative, de sorte qu'il en ressort une antithèse qui oppose la première partie à la deuxième. Dans le premier, la mise en avant des adjectifs desgrazados et máis desgrazados au début de chacune des séquences permet de relever les conséquences dramatiques de la perte d'une mère, alors que dans le deuxième on thématise l'évènement de la mort morte de pai - morte de nai. En outre, dans le proverbe a), les deux propositions présentent une structure syntaxique parallèle, avec une rime en -ai, ce qui contribue à la mémorisation et la transmission de la parémie. Dans le proverbe b), la mise en parallèle des deux propositions est sous-entendue, étant donné que dans la deuxième partie on y omet desfai casa, en le remplaçant par l'adverbe si. On constate dans celui-ci l'emploi de la métaphore desfacer unha casa qui fait allusion à détruire une famille, dans le sens de la laisser sans guide, sans protection, sans soutien. Finalement, l'absence de déterminants dans les propositions nominales insuffle une plus grande expressivité aux concepts impliqués. À noter aussi la présence d'une allitération en $-a j$.

\section{Conclusion}

Les proverbes constituent une partie fondamentale de la représentation sociale des peuples, de leurs coutumes et de leurs croyances, et permettent donc une analyse fiable de la réalité collective et de son évolution. C'est dans cette profonde interdépendance de la langue et de la société que nous avons entrepris la recherche de proverbes qui font l'éloge de la femme. Assurément, bien plus connus et bien plus nombreux sont ceux qui mettent en avant des traits négatifs, et qui sont issus cette vision phallocratique de l'idéologie traditionnelle de la figure féminine et de son rôle au sein de la société. Cependant, « ce n'est pas parce qu'ils sont nombreux à avoir tort qu'ils ont raison » (Bisignani, Isaert, 2014 : 131). En effet, l'image stéréotypée de la femme se réalise généralement d'un point de vue masculin, chargé de connotations sexistes, parfois même hostiles comme dans « Il est permis de battre sa femme, mais il ne faut pas l'assommer », dans le but de maintenir l'ordre social patriarcal. Ce genre de proverbes ont été véhiculés depuis l'Antiquité grecque jusqu'à nos jours, comme l'exemple, issu des préceptes

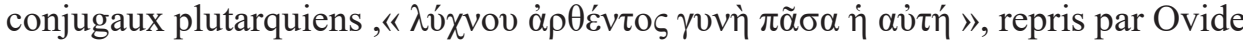


dans L'art d'aimer et arrivé dans la plupart des langues romanes avec des variantes plus ou moins proches, telles qu'en français « La nuit, il n'y a point de femme laide », "À la chandelle, la chèvre semble demoiselle », en espagnol «A la luz de la vela no hay mujer fea », "A la vela, la burra parece doncella ", «A la luz de la candela, toda rústica parece bella » ou en galicien « De noite non hai muller fea », mais aussi en italien « A lume spento è pari ogni bellezza », en portugais « De noite, à candeia, parece bonita a feia », « No escuro, tanto faz a rainha como a negra da cozinha », et même en allemand « Bei Nacht sind alle Weiber gleich » et en anglais "When the candles are out, all women are fair » (Pamies Beltran, $2011: 33)$.

En tant que linguiste, nous entendons qu'il est de notre responsabilité de recueillir les proverbes en usage, ainsi que ceux tombés en désuétude, pour contribuer ainsi à la mise en lumière de ces pilules de sagesse et mener à bien cette approche comparative. Mais, dans la lignée de la théorie de la relativité linguistique et dans cet esprit de promouvoir la sagesse navajo résumée dans "Choisis bien tes mots, car ce sont eux qui créent le monde qui t'entoure », nous avons opté pour dénicher ces quelques autres proverbes qui, contrairement, à cette grande majorité, offrent une image positive de la femme, en les comparant par la suite dans les trois langues.

Ainsi, nous avons constaté que, malgré des équivalences qui peuvent se produire, tantôt dans la forme, tantôt dans le sens, celles-ci sont rarement pleines entre les proverbes français, espagnols et galiciens. Les principaux éléments discordants se rapportent à une plus grande longueur des proverbes en langue française, partiellement due aux connotations religieuses y figurant, mais aussi à une expression généralement plus gracieuse, moins abrupte, ainsi qu'à la perdurance d'un plus grand nombre de structure fossilisées et à l'emploi de structures bimembres débutant par une subordonnée relative en fonction de sujet. Quant à la thématique de la femme, on observe également dans ces derniers un plus grand nombre de parémies aimables et bienveillantes à son égard, tout en dédaignant l'importance accordée à la beauté. Les proverbes galiciens partagent également ce dernier aspect, bien que leur expression soit beaucoup plus concise, voire rude, avec bon nombre d'allusions, explicites ou sous-entendues, au monde rural, ainsi qu'au système matrilinéaire hérité des Celtes. Leur brièveté et sobriété garantit généralement leur mémorisation et transmission au fil du temps, éludant ainsi d'autres recours stylistiques comme la rime ou la métaphore.

Car, bien que l'emploi des proverbes dans la langue se soit généralement affaibli, on continue à les utiliser et il arrive même qu'on y ait recours à des fins publicitaires, parfois sous des formes originales, d'autres de manière infidèle, enfantant ainsi des antiparémies, des faux proverbes au sens amoral ou opposé à l'original, comme un clin d'œil aux consommateurs. La possible interaction de ces unités phraséologiques chez les interlocuteurs est analysée par l'ethnolinguistique et la psycholinguistique, en fonction des tendances du moment, comme l'actuel 
mouvement féministe, de sorte que leur ajustement à la société permet bien souvent l'adoption de ces slogans, qui viennent, avec le temps, rétroalimenter nos répertoires parémiologiques, comme le dorénavant fameux « Parce que je le vaux bien », créé par L’Oréal.

\section{Bibliographie}

ÁLVAREZ DE LA GRANJA, María (2003), « Proposta de clasificación semántico-funcional das unidades fraseolóxicas galegas », Cadernos de fraseoloxía galega, $n^{\circ}$ 4, p. 1-26

ANSCOMBRE, Jean-Claude (1994), «Proverbes et formes proverbiales : valeur évidentielle et argumentative $»$, Langue française, $n^{\circ} 102$, p. 95-106

ARNAUD, Pierre (1992), « Réflexions sur le refrain », Cahiers de Lexicologie, n 59/2, p. 6-27

BELAMRI, Rabah (1986), Proverbes et dictons algériens, Paris, L'Harmattan

BISIGNANI, Thomas, ISAERT, Benoit (2014), Proverbes à la con-Mieux vaut être bon à rien que mauvais à tout, Paris, First

CLAVER ZOUOGBO, Jean-Philippe (2009), Le proverbe entre langues et cultures : une étude de linguistique confrontative, Berne, Peter Lang

CONDE TARRÍO, Germán (1998), « La verdad en el refranero : los refranes meteorológicos gallegos », Paremia, n' 7, p. 61- 68

CORPAS PASTOR, Gloria (1996), Manual de fraseología española, Madrid, Gredos

DESALMAND, Paul, STALLONI, Yves (2013), Proverbes expliqués, Paris, Éditions du Chêne

FERNÁNDEZ PONCELA, Anna María (2015), Amor, matrimonio y etapas de la vida. Discurso didácticomoral y consejos prácticos en el refranero español, Valladolid, Fundación Joaquín Díaz

GARCÍA-PAGE, Mario (2008), Introducción a la fraseología española, Barcelona, Anthropos

Génesis: II, 24

GŁOWICKA, Monika (2007), «Visión de la familia en los refranes españoles », Estudios Hispánicos, $\mathrm{n}^{\circ} 15$, p. 265-275

GROSS, Maurice (1982), «Une classification des phrases “figées” du français », Revue québécoise de linguistique, $\mathrm{n}^{\circ} 11, \mathrm{p} .151-185$

INSTITUTO CERVANTES (1997), Refranero multilingüe, Madrid. (https://cvc.cervantes.es/ lengua/refranero/Busqueda.aspx, consulté le 09 octobre 2019)

KLEIBER, Georges (1988), "Sur la définition du proverbe », Europhras 88. Phraséologie contrastive, p. 233-252

LADMIRAL, Jean-René (1999), "Sur la philosophie de la culture impensée de la traduction », Parcours, passages et paradoxes interculturels, p. 141-164

LÁZARO CARRETER, Fernando (1980), Estudios de lingüística, Barcelona, Crítica

Les Proverbes de Salomon XVIII, (22)

LORENZO FERNÁNDEZ, Xaquín (1983), Refraneiro Galego, Vigo, Castrelos

MÉNAGE, Gilles (1654), Menagiana ou bons mots, rencontres agréables, pensées judicieuses et observations curieuses, Paris, Pierre Delaulne. (https://reader.digitale-sammlungen.de/de/fs1/ object/display/bsb11342763_00006.html, consulté le 05 octobre 2019)

MOREIRAS SANTISO, Xosé (1978), Os mil e un refráns galegos da muller, Lugo, Alvarellos

NORRICK, Neal (1985), How proverbs mean: semantic studies of English proverbs, Berlin, Mouton

OULD EBNOU, Mohamedou, OULD MOHAMEDEN, Moussa (2008), Encyclopédie de la Culture Populaire Mauritanienne - Contes et Proverbes de Mauritanie, vol. III, Paris, L'Harmattan

PAMIES BELTRÁN, Antonio, LUQUE DURÁN, Juan de Dios, FERNÁNDEZ MARTÍN, Patricia (2011), Paremiología y herencia cultural, Granada, Granada Lingüística 
QUITARD, Pierre-Marie (1861), Proverbes sur les femmes, l'amitié, l'amour et le mariage, Paris, Garnier Frères. (https://gallica.bnf.fr/ark:/12148/bpt6k5462189b.texteImage, consulté le 27 septembre 2019)

RODEGEM, François-Marie (1973), « Un problème de terminologie : les locutions sentencieuses », Cahiers de linguistique de Louvain, $\mathrm{n}^{\circ} 1 / 5$, p. 678-703

RODRIGUEZ FERREIRO, Verónica (2017), Refrans \& Proverbes. (http://refrans- proverbes.com, consulté le 20 septembre 2019)

SCHIPPER, Mineke (2008), Belle femme, gros ennuis - Les femmes en proverbes du monde entier, Paris, Philippe Rey

SESTO LÓPEZ, Xosé (1976), Refraneiro da muller, Vigo, Castrelos

TÊTU, Michel (1992), La Francophonie : Historie, problématique et perspectives, Chamonix, Guérin

THÉROND, Maurice (1972), Du tac au tac: formules, réflexes et images de la conversation française actuelle, Paris, Marcel Didier

ZAMORA MOSQUERA, Federico (1972), Refráns e ditos populares galegos, Vigo, Galaxia

ZULUAGA OSPINA, Alberto (1975), «La fijación fraseológica », Thesaurus, XXX, n² 2, p. 225248

Véronica Rodriguez Ferreiro - doctorante en Linguistique française à l'Université de SaintJacques de Compostelle. Sa thèse porte sur l'« Évolution du féminin dans les désignants de professions et discours politiquement correct » et fait partie de l'équipe de travail du projet de recherche «El eufemismo en la prensa francófona actual y su dimensión polifónica. Perspectivas dialógicas ». Elle est professeure-tutrice de français à l'Université Nationale d'Éducation à Distance et dans l'Enseignement secondaire. 